\title{
Solving a Cauchy problem for a 3D elliptic PDE with variable coefficients by a quasi-boundary- value method
}

\author{
Xiaoli Feng and Lars Eldén
}

\section{Linköping University Post Print}

\section{Tweet}

N.B.: When citing this work, cite the original article.

Original Publication:

Xiaoli Feng and Lars Eldén, Solving a Cauchy problem for a 3D elliptic PDE with variable coefficients by a quasi-boundary-value method, 2014, Inverse Problems, (30), 1, 015005.

http://dx.doi.org/10.1088/0266-5611/30/1/015005

Copyright: Institute of Physics: Hybrid Open Access http://www.iop.org/

Postprint available at: Linköping University Electronic Press http://urn.kb.se/resolve?urn=urn:nbn:se:liu:diva-103280 


\title{
Solving a Cauchy Problem for a 3D Elliptic PDE with Variable Coefficients by a Quasi-Boundary-Value Method*
}

\author{
Xiaoli Feng ${ }^{1 \dagger}$ and Lars Eldén ${ }^{2}$ \\ 1. Department of Mathematics, Xidian University, Xi'an 710071, People's Republic of China \\ 2. Department of Mathematics, Linköping University, SE-581 83 Linköping, Sweden
}

\begin{abstract}
An ill-posed Cauchy problem for a 3D elliptic PDE with variable coefficients is considered. A well-posed quasi-boundary-value (QBV) problem is given to approximate it. Some stability error estimates are given. For the numerical implementation, a large sparse system is obtained from the discretizing the QBV problem using finite difference method (FDM). A LeftPreconditioner Generalized Minimum Residual (GMRES) method is used to solve the large system effectively. For the preconditioned system, a fast solver using the Fast Fourier Transform (FFT). Numerical results show that the method works well.

Key words. Elliptic equation; Ill-Posed; Cauchy problem; Finite difference method; Quasi-boundary-value method; Left-Preconditioned GMRES; Fast solver; Variable coefficients; Three dimensions; Fast Fourier Transform
\end{abstract}

\section{Introduction}

Let $\Omega=\left\{\left(y_{1}, y_{2}\right) \mid 0<y_{1}<1,0<y_{2}<1\right\}$ and consider the following ill-posed Cauchy problem for an elliptic PDE,

$$
\begin{array}{lr}
\left(a u_{x}\right)_{x}+\left(b u_{y_{1}}\right)_{y_{1}}+\left(c u_{y_{2}}\right)_{y_{2}}=f\left(x, y_{1}, y_{2}\right), & \left(x, y_{1}, y_{2}\right) \in(0,1) \times \Omega, \\
u\left(x, y_{1}, y_{2}\right)=\phi\left(x, y_{1}, y_{2}\right), & x \in[0,1],\left(y_{1}, y_{2}\right) \in \partial \Omega, \\
u\left(0, y_{1}, y_{2}\right)=\psi\left(y_{1}, y_{2}\right), & \left(y_{1}, y_{2}\right) \in \bar{\Omega}, \\
u_{x}\left(0, y_{1}, y_{2}\right)=g\left(y_{1}, y_{2}\right), & \left(y_{1}, y_{2}\right) \in \bar{\Omega},
\end{array}
$$

${ }^{*}$ The project is supported by the National Natural Science Foundation of China (No. 11171136), the Fundamental Research Funds for the Central Universities (No. K50511700002) and the China Postdoctoral Science Foundation (No. 2012M521742).

${ }^{\dagger}$ E-mails: xiaolifeng@xidian.edu.cn, lars.elden@liu.se 
where the coefficients are assumed to be piecewise differentiable satisfying

$$
\begin{array}{lll}
a\left(x, y_{1}, y_{2}\right) \geq a_{0}>0, & b\left(x, y_{1}, y_{2}\right) \geq b_{0}>0, & c\left(x, y_{1}, y_{2}\right) \geq c_{0}>0 \\
-a_{1} \leq a_{x} \leq a_{2}, & -b_{1} \leq b_{x}, & -c_{1} \leq c_{x}
\end{array}
$$

The constants $a_{1}, b_{1}$, and $c_{1}$ are assumed to be non-negative. The problem is to compute $u\left(x, y_{1}, y_{2}\right)$ numerically for $0<x \leq 1$.

The Cauchy problem for an elliptic equation is a classical ill-posed problem and occurs in several important applications, such as inverse scattering $[17,37]$, electrical impedance tomography [10], optical tomography [7], and thermal engineering [23]. The topic is treated in several monographs [16,36,37,41,42,45], and in numerous papers, see $[1,3,6,8,9,18,24,25,34,47,48,51,57,61]$ and the references therein. Even if some of the theoretical investigations are quite general, the numerical procedures proposed are typically for the two dimensional case and often only valid for the problem with constant coefficients. To our knowledge there are no papers in the literature that treat the numerical solution of elliptic Cauchy problems in three dimensions with all three coefficients depending on $\left(x, y_{1}, y_{2}\right)$. In this paper, we propose to use a quasi-boundary-value [QBV] method $^{1}[2]$ to regularize the problem (1.1)-(1.5) as follows:

$$
\begin{array}{lr}
\left(a v_{x}\right)_{x}+\left(b v_{y_{1}}\right)_{y_{1}}+\left(c v_{y_{2}}\right)_{y_{2}}=f^{\delta}\left(x, y_{1}, y_{2}\right), & \left(x, y_{1}, y_{2}\right) \in(0,1) \times \Omega, \\
v\left(x, y_{1}, y_{2}\right)=\phi^{\delta}\left(x, y_{1}, y_{2}\right), & x \in[0,1],\left(y_{1}, y_{2}\right) \in \partial \Omega, \\
v\left(0, y_{1}, y_{2}\right)=\psi^{\delta}\left(y_{1}, y_{2}\right), & \left(y_{1}, y_{2}\right) \in \bar{\Omega}, \\
v_{x}\left(0, y_{1}, y_{2}\right)+\alpha v\left(1, y_{1}, y_{2}\right)=g^{\delta}\left(y_{1}, y_{2}\right), & \left(y_{1}, y_{2}\right) \in \bar{\Omega},
\end{array}
$$

where the noisy data satisfy

$$
\left\|f^{\delta}-f\right\|+\left\|\phi^{\delta}-\phi\right\|+\left\|\psi^{\delta}-\psi\right\|+\left\|g^{\delta}-g\right\| \leq \delta .
$$

The notation $\|\cdot\|$ denotes $L^{2}-$ norm. For the numerical solution of well-posed problems for general elliptic PDE's in three dimensions usually only iterative methods are effective. For nonselfadjoint problems the Generalized Minimum Residual method (GMRES) [53] is a standard iterative method. However, without a preconditioner the GMRES method converges slowly or even does not work.

Our proposed method is the following. After discretization (1.6)-(1.10) is reduced to a nonsymmetric linear system, with a large and sparse matrix. We solve it using preconditioned GMRES. As preconditioner we use the discretization of a nearby QBV equation where the variable coefficients have been replaced by constants. Such a problem can be solved very efficiently, using Fast Fourier Transform (FFT) techniques. We demonstrate that problems with half a million unknowns can be solved using MATLAB in about 15 iterations and in less than 90 seconds on a standard PC.

\footnotetext{
${ }^{1}$ Also called the modified boundary method, or non-local boundary value problem method.
} 
To motivate our choice of solution procedure we first note that for partial differential equations with variable coefficients one cannot obtain fundamental solutions, and therefore classical regularization methods depending on the reformulation as an integral equation, such as Tikhonov method, cannot be used. Thus it is necessary to keep the equation in PDE form. We then recall that for well-posed PDE's iterative procedures are routinely used. This approach has also been applied to the Cauchy problem, where a certain energy functional is minimized [6]; unfortunately, in each iteration of that procedure four well-posed elliptic equations would have to be solved over the whole three-dimensional domain. Some iterative methods, e.g. based on the alternating procedure of [40], often converge very slowly. Another approach [25] based on the Krylov subspace method for the cylindrical case requires the solution of only one two-dimensional problem at each iteration. However, it can not deal with the problem (1.1)-(1.5) with variable coefficient $a\left(x, y_{1}, y_{2}\right)$.

QBV-type methods have been used before for ill-posed problems [4, 5, 21, 22, $32-34,43,54,58-60]$. In [27], we applied the method to a Cauchy problem for an elliptic equation, and studied its stability. We also made a preliminary numerical implementation for a slightly less general problem than the one in the present paper. Now we further develop the implementation from [27] and demonstrate that it is an efficient algorithm for solving the general problem (1.1)-(1.5) with variable coefficients.

Preconditioned GMRES is widely used not only for well-posed problems, see e.g. [12,52], but also for ill-posed problems $[11,13-15,31,38,39]$. The regularizing properties of preconditioned Krylov methods were investigated in [30]. To the authors' knowledge, preconditioned GMRES applied to an ill-posed problem has mostly been used for the discretization of an integral equation of the first kind, stabilized by Tikhonov regularization. However, in [49,50] an ill-posed parabolic problem was solved using GMRES with a singular preconditioner, see also [26]. In the present paper, we approximate the ill-posed elliptic PDE by a well-posed QBV problem, and then we use preconditoned GMRES to solve the linear system of equations obtained by discretizing the well-posed problem.

The paper is organized as follows. First, in Section 2, we give an explicit stability result for the Cauchy problem. Then we describe in Section 3 how the QBV problem (1.6)-(1.10) can be discretized and solved iteratively using GMRES. However, as in the well-posed case, a preconditioner is necessary in order for GMRES to be efficient. Our preconditioner is the same QBV method applied to a nearby Cauchy problem with constant coefficients. In Section 3.3, we describe a fast solver for problem (1.6)-(1.10) with the constant coefficients. The problem of choosing the regularization parameter is discussed in Section 4. Finally, in Section 5, we discuss the numerical implementation and give some experiments that demonstrate the efficiency of the proposed method. 


\section{An Explicit Stability Result}

It is well known that the Cauchy problem for an elliptic equation is severely illposed. General stability estimates are given in, e.g., [3,37]; see also [16,34]. In the case of a rectangular geometry, more concrete results can be found [24]. In the numerical solution of a mathematical problem perturbations due to discretization and round-off in the floating point arithmetic are inevitably introduced. Therefore, it is necessary to have explicit and concrete stability results for the underlying mathematical problem [24]. By explicit we here mean estimates, given under the assumption that certain regularity conditions are satisfied, where the quantities are expressed in terms of the data and coefficients of the problem. Actually, such conditional stability results imply not only the uniqueness of the solutions of the problems but also the convergence rate of the regularized solutions [19].

We here give a stability result of Hölder type for the following special problem, which is inspired by results in $[24,44-46]$, and which is a slight generalization of that in [24].

$$
\begin{array}{lr}
\left(a u_{x}\right)_{x}+\left(b u_{y_{1}}\right)_{y_{1}}+\left(c u_{y_{2}}\right)_{y_{2}}=0, & \left(x, y_{1}, y_{2}\right) \in(0,1) \times \Omega, \\
u\left(x, y_{1}, y_{2}\right)=0, & x \in[0,1],\left(y_{1}, y_{2}\right) \in \partial \Omega, \\
u\left(0, y_{1}, y_{2}\right)=0, & \left(y_{1}, y_{2}\right) \in \bar{\Omega}, \\
u_{x}\left(0, y_{1}, y_{2}\right)=g\left(y_{1}, y_{2}\right), & \left(y_{1}, y_{2}\right) \in \bar{\Omega} .
\end{array}
$$

Before giving the stability result, we present some notations. Define the family of rectangular regions, parameterized by

$$
D_{\beta}=\left\{\left(x, y_{1}, y_{2}\right) \mid 0 \leq x \leq \beta,\left(y_{1}, y_{2}\right) \in \Omega\right\}, \quad 0 \leq \beta \leq 1 .
$$

Set

$$
K_{1}=\widetilde{C_{0}}+4 \lambda \text { and } K_{2}=2 \lambda\left(2 \lambda+\widetilde{C_{0}}\right),
$$

where the constant $\lambda$ satisfies

$$
\lambda \geq \max \left(\frac{a_{2}}{2 a_{0}}, \frac{a_{1}}{a_{0}}\right),
$$

and

$$
\widetilde{C_{0}}=\max \left(\frac{a_{1}}{a_{0}}, \frac{b_{1}}{b_{0}}, \frac{c_{1}}{c_{0}}\right) .
$$

We introduce also the function

$$
\nu(\beta)=\frac{1-e^{-K_{1} \beta}}{1-e^{-K_{1}}} .
$$


Theorem 1 Assume that the two functions $u_{1}$ and $u_{2}$ satisfy problem (2.1)-(2.4) with different data $g_{i}, i=1,2$, and the boundedness assumptions

$$
\int_{D_{1}} a u_{i}^{2} d x d y_{1} d y_{2} \leq E_{2}, \quad i=1,2,
$$

for some constant $E_{2}$. If the data error is bounded

$$
\int_{0}^{1} \int_{0}^{1} a\left(0, y_{1}, y_{2}\right)\left(g_{1}\left(y_{1}, y_{2}\right)-g_{2}\left(y_{1}, y_{2}\right)\right)^{2} d y_{1} d y_{2} \leq \widetilde{\delta}
$$

then, for $0 \leq \beta \leq 1$,

$$
\int_{D_{\beta}} a\left(u_{1}-u_{2}\right)^{2} d x d y_{1} d y_{2} \leq 4 e^{(-\beta+\nu(\beta)) K_{2} / K_{1}} E_{3}^{\nu(\beta)} \widetilde{\delta}^{1-\nu(\beta)},
$$

where $E_{3}=2\left(\lambda+\left(1+a_{1} / a_{0}\right) / 2\right) E_{2}+\widetilde{\delta}$.

Proof. A sketch of the proof is given in the appendix. Although the boundary condition is different, there is no essential difference from that in [24].

Remark 1 In fact, the results hold also for a general domain $\Omega$. For the problem nonhomogeneous Dirichlet data, we can also obtain the same result as in Theorem 1. For the Cauchy problem (1.1)-(1.5) with nonhomogeneous Cauchy and boundary data, we can use the linearity of the problem and divide it into an ill-posed problem and a well-posed one to obtain the stability estimate [24]. We can also get the similar result by using the technique of [44]. Unfortunately it is not easy to derive an error estimate for the QBV solution. This will be a topic of our future research.

Remark 2 Note that the stability estimate (2.8), depending explicitly on the coefficients, allows us to draw the conclusion that if $a_{x}$ is negative then the stability becomes worse than in the case of positive $a_{x}$, in the sense that $E_{3}$ becomes larger in the first case.

\section{Solving the QBV Problem by GMRES}

In this section, we will consider the solution of the QBV problem (1.6)-(1.10) with variable coefficients by preconditioned GMRES. First we describe the finite difference discretization of the problem. In our presentation it is essential to use finite differences, since in that case the action of our preconditioner can be implemented very efficiently. However, also a finite element discretization could be used, as long as one can find a good enough preconditioner.

Before using the left-preconditioned GMRES method, a suitable value of the regularization parameter $\alpha$ must be chosen. We discuss this in Section 4 . 


\subsection{Discretization}

We here describe the discretization of the problem (1.6)-(1.10) using the finite difference method (FDM) with central differences.

Let the interval $[0,1]$ in the $x$ direction be divided into $N_{x}$ segments of length $h_{x}$, and, similary, the intervals in the $y_{1}$ and $y_{2}$ directions be divided into $N_{y}$ segments of length $h_{y}$. Then the coefficient matrix $\widetilde{A}$ has dimension $N_{x}\left(N_{y}-\right.$ $1)^{2} \times N_{x}\left(N_{y}-1\right)^{2}$. In detail, the PDE (1.6) is discretized

$$
\begin{aligned}
& a\left(i-\frac{1}{2}, j, k\right) v(i-1, j, k)+r b\left(i, j-\frac{1}{2}, k\right) v(i, j-1, k) \\
& +r c\left(i, j, k-\frac{1}{2}\right) v(i, j, k-1)-\left\{a\left(i-\frac{1}{2}, j, k\right)+a\left(i+\frac{1}{2}, j, k\right)\right. \\
& \left.+r\left[b\left(i, j-\frac{1}{2}, k\right)+b\left(i, j+\frac{1}{2}, k\right)\right]+r\left[c\left(i, j, k-\frac{1}{2}\right)+c\left(i, j, k+\frac{1}{2}\right)\right]\right\} v(i, j, k) \\
& +r b\left(i, j+\frac{1}{2}, k\right) v(i, j+1, k)+r c\left(i, j, k+\frac{1}{2}\right) v(i, j, k+1) \\
& +a\left(i+\frac{1}{2}, j, k\right) v(i+1, j, k)=h_{x}^{2} f^{\delta}(i, j, k),
\end{aligned}
$$

with $r=h_{x}^{2} / h_{y}^{2}$. For the quasi-boundary condition (1.9) we get

$$
\frac{v(1, j, k)-v(-1, j, k)}{2 h_{x}}+\alpha v\left(N_{x}, j, k\right)=g^{\delta}(j, k) .
$$

The linear system becomes

$$
\widetilde{A} V=F
$$

where $\widetilde{A}$ has the structure

$$
\widetilde{A}=\left(\begin{array}{ccccc}
\left(A_{0}+A_{1}\right) / 2 & 0 & \ldots & \ldots & \alpha h_{x} A_{0} \\
B_{1} & A_{2} & 0 & & \\
A_{2} & B_{2} & A_{3} & & \\
& \ddots & \ddots & \ddots & \\
0 & \ldots & A_{N_{x}-1} & B_{N_{x}-1} & A_{N_{x}}
\end{array}\right),
$$

with block-diagonal matrices $A_{i}$,

$$
A_{i}=\operatorname{diag}\left(\mathcal{A}_{i}^{1}, \mathcal{A}_{i}^{2}, \ldots, \mathcal{A}_{i}^{N_{y}-1}\right) .
$$

The diagonal blocks are given by

$$
\mathcal{A}_{i}^{j}=\operatorname{diag}\left(a(i-1 / 2, j, 1), a(i-1 / 2, j, 2), \ldots, a\left(i-1 / 2, j, N_{y}-1\right)\right),
$$

while the matrices $B_{i}$ are block-tridiagonal

$$
B_{i}=\operatorname{tridiag}\left(\mathcal{B}_{i}^{j}, \mathcal{C}_{i}^{j}, \mathcal{B}_{i}^{j}\right),
$$


with tridiagonal blocks

$\mathcal{C}_{i}^{j}=\operatorname{tridiag}(r c(i, j, k+1 / 2), d(i, j, k), r c(i, j, k+1 / 2)), \quad \mathcal{B}_{i}^{j}=\operatorname{diag}(r b(i, j+1 / 2, k))$,

where

$$
\begin{aligned}
d(i, j, k)= & -\left\{a\left(i-\frac{1}{2}, j, k\right)+a\left(i+\frac{1}{2}, j, k\right)+r\left[b\left(i, j-\frac{1}{2}, k\right)+b\left(i, j+\frac{1}{2}, k\right)\right]\right. \\
& \left.+r\left[c\left(i, j, k-\frac{1}{2}\right)+c\left(i, j, k+\frac{1}{2}\right)\right]\right\} .
\end{aligned}
$$

The structure of the linear system (3.3) is similar to that of a discretization of a 3D selfadjoint elliptic boundary value problem, with the important exception that here the matrix is nonsymmetric, due to the quasiboundary condition. In general, linear systems arising from 3D problems with variable coefficients can be solved efficiently only using iterative methods, see, [52] for an overview. Since $\widetilde{A}$ is non-symmetric, we use the Generalized Minimum Residual Method (GMRES) [53] (described in the following subsection).

However, it turns out that GMRES without preconditioning does not converge fast enough.

Example 1 Consider a nonhomogeneous equation in the unit cube with $a\left(x, y_{1}, y_{2}\right)=$ $x+y_{1}+y_{2}+1, b\left(x, y_{1}, y_{2}\right)=x y_{1} y_{2}+1, c\left(x, y_{1}, y_{2}\right)=x\left(y_{1}+y_{2}\right)+1$, and an exact solution $u\left(x, y_{1}, y_{2}\right)=\sin \left(x+y_{1}+y_{2}\right)$, i.e.,

$$
\begin{cases}\left(a u_{x}\right)_{x}+\left(b u_{y_{1}}\right)_{y_{1}}+\left(c u_{y_{2}}\right)_{y_{2}}=f\left(x, y_{1}, y_{2}\right), & \left(x, y_{1}, y_{2}\right) \in(0,1)^{3}, \\ u\left(x, 0, y_{2}\right)=\sin \left(x+y_{2}\right), & \left(x, y_{2}\right) \in[0,1]^{2}, \\ u\left(x, 1, y_{2}\right)=\sin \left(x+1+y_{2}\right), & \left(x, y_{2}\right) \in[0,1]^{2}, \\ u\left(x, y_{1}, 0\right)=\sin \left(x+y_{1}\right), & \left(x, y_{1}\right) \in[0,1]^{2}, \\ u\left(x, y_{1}, 1\right)=\sin \left(x+y_{1}+1\right), & \left(x, y_{1}\right) \in[0,1]^{2}, \\ u\left(0, y_{1}, y_{2}\right)=\sin \left(y_{1}+y_{2}\right), & \left(y_{1}, y_{2}\right) \in[0,1]^{2}, \\ u_{x}\left(0, y_{1}, y_{2}\right)=\cos \left(y_{1}+y_{2}\right), & \left(y_{1}, y_{2}\right) \in[0,1]^{2},\end{cases}
$$

The right hand side becomes $f\left(x, y_{1}, y_{2}\right)=\left(1+x y_{2}+x\right) \cos \left(x+y_{1}+y_{2}\right)-(x+$ $\left.y_{1}+y_{2}+x y_{1} y_{2}+x\left(y_{1}+y_{2}\right)+3\right) \sin \left(x+y_{1}+y_{2}\right)$.

For a finite difference discretization with 49 unknowns in each $y$-direction and 50 steps in $x$ we get a $120050 \times 120050$ matrix $\widetilde{A}$. We chose the regularization parameter $^{2} \alpha=0.0003$. The GMRES method applied to the $Q B V$ problem did not converge in 3430 iterations. Note that here we used restarted GMRES, with restart after every 30 iterations (for restarted GMRES, see the next subsection).

Apparently, the reason for the slow convergence is that even if the ill-posed problem is regularized, it is still quite ill-conditioned. In [27] the GMRES method

\footnotetext{
${ }^{2}$ Here the regularization parameter was chosen by the a-priori rule in [27].
} 
did not work even for the two dimensional problem with constant coefficients, and we therefore asked whether it is possible to solve the linear equations obtained in the $Q B V$ method using preconditioned GMRES. We there successfully constructed a preconditioner for the $2 \mathrm{D}$ problem based on approximation by a circulant matrix. In the following subsection we first give a quick introduction to preconditioned GMRES, and then show how to construct a preconditioner for the variable coefficient case that leads to fast convergence also in the $3 \mathrm{D}$ case.

\subsection{GMRES with Preconditioning}

Preconditioning is a key ingredient for the success of Krylov subspace methods for linear systems [52, Chapter 9]. The main purpose of using a preconditioner in the iterative solution of a linear system of equations is to reduce the number of iterations and, at the same time, the computing time. In general, the efficiency of iterative techniques depends on the quality of the preconditioner much more than the particular Krylov subspace method used. In the case of the GMRES, or other nonsymmetric iterative solvers, three options for applying the preconditioning operation (i.e., left, split and right preconditioning) are available. The left-preconditioned GMRES algorithm is stated in Figure $1^{3}$. In statements 4-7

Figure 1: ALGORITHM: GMRES with Left Preconditioning

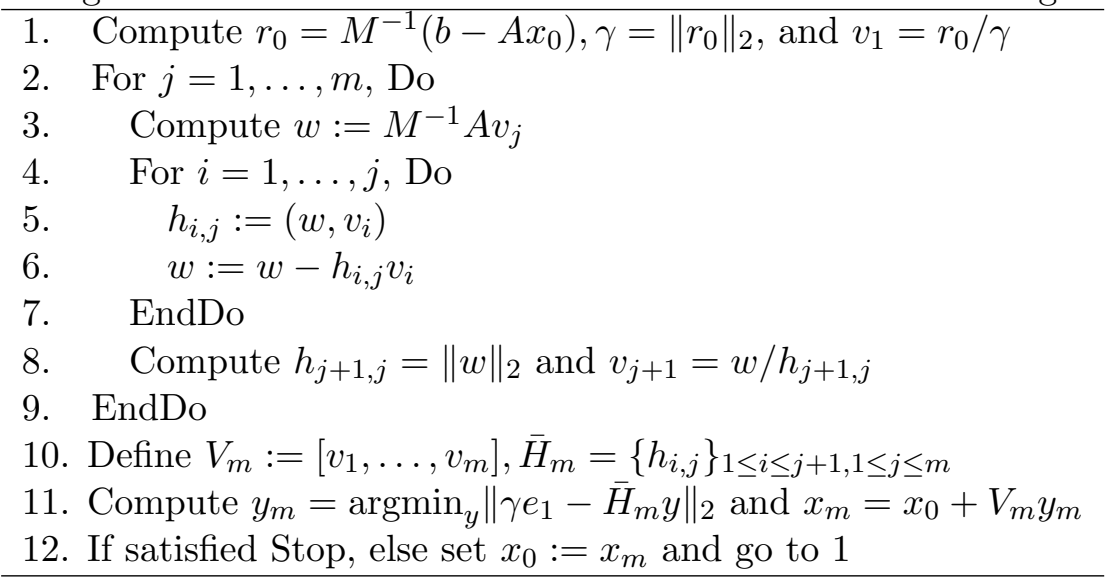

the newly computed Krylov basis vector $w$ is orthogonalized against $v_{1}, \ldots, v_{j}$. In cases when the algorithm does not converge very quickly, the storage of the $v_{j}$ vectors can become prohibitive, due to memory restrictions and the work for orthogonalization. In that case, one often uses restarted GMRES: after a predefined number of steps, all basis vectors are discarded except the most recently computed one, and the procedure is restarted. In our context restarted GMRES was only

\footnotetext{
${ }^{3}$ Of course, unpreconditioned GMRES corresponds to putting $M$ equal to the identity.
} 
used in Example 1.

As preconditioner we use the matrix for the QBV method given by the problem with the constant coefficients, chosen as the mean values of the respective variable coefficients. It turns out that the linear system with this matrix can be solved very efficiently using a direct solver.

\subsection{Fast Solver for the Constant Coefficient Case}

The QBV problem for the constant coefficient case is

$$
\begin{array}{lr}
a v_{x x}+b v_{y_{1} y_{1}}+c v_{y_{2} y_{2}}=f^{\delta}\left(x, y_{1}, y_{2}\right), & \left(x, y_{1}, y_{2}\right) \in(0,1) \times \Omega, \\
v\left(x, y_{1}, y_{2}\right)=\phi^{\delta}\left(x, y_{1}, y_{2}\right), & x \in[0,1],\left(y_{1}, y_{2}\right) \in \partial \Omega, \\
v\left(0, y_{1}, y_{2}\right)=\psi^{\delta}\left(y_{1}, y_{2}\right), & \left(y_{1}, y_{2}\right) \in \bar{\Omega}, \\
v_{x}\left(0, y_{1}, y_{2}\right)+\alpha v\left(1, y_{1}, y_{2}\right)=g^{\delta}\left(y_{1}, y_{2}\right), & \left(y_{1}, y_{2}\right) \in \bar{\Omega},
\end{array}
$$

where the regularization parameter $\alpha$ is chosen the same as for non-constant coefficients. We will now briefly describe a fast solver for the discretization of this PDE problem, based on the FFT, in a manner analogous to that when solving well-posed problems for Poisson's equation $[35,56]$.

If we discretize (3.5)-(3.8) in exactly the same way as in Section 3.1, we obtain a linear system $A U=F$ of the same dimension as $\widetilde{A}$ in (3.3), with

$$
A=\left(\begin{array}{ccccc}
a I & 0 & \ldots & \ldots & -\alpha h_{x} a I \\
T & a I & 0 & & \\
a I & T & a I & & \\
& \ddots & \ddots & \ddots & \\
0 & \ldots & a I & T & a I
\end{array}\right)
$$

where the block $T$ has the structure

$$
T=T_{2} \otimes I+I \otimes T_{1},
$$

with tridiagonal matrices $T_{1}, T_{2}$ ( $\otimes$ denotes the Kronecker product).

We can block-diagonalize the system $A U=F$ by discrete trigonometric transforms. Let $S$ be the $\left(N_{y}-1\right) \times\left(N_{y}-1\right)$ discrete sine transform matrix (DST) $S_{i j}=\sin \left(\pi i j / N_{y}\right)$. Then $T$ can be diagonalized by the following transformation (which is equivalent to a $2 \mathrm{D}$ DST [20]),

$$
\left(S^{*} \otimes S^{*}\right) T(S \otimes S)=\left(S^{*} T_{2} S\right) \otimes I+I \otimes\left(S^{*} T_{1} S\right)=\Lambda_{2} \otimes I+I \otimes \Lambda_{1}:=\Lambda ;
$$

(here $S^{*}$ is the conjugate transpose of $S$ ). The matrices $\Lambda_{1}$ and $\Lambda_{2}$ are diagonal with explicit expressions for the elements [55, p. 113]. After this transformation 
the matrix $A$ is replaced by

$$
\left(\begin{array}{ccccc}
a I & 0 & \ldots & \ldots & -\alpha h_{x} a I \\
\Lambda & a I & 0 & & \\
a I & \Lambda & a I & & \\
& \ddots & \ddots & \ddots & \\
0 & \ldots & a I & \Lambda & a I
\end{array}\right) .
$$

Since the linear system with the matrix (3.12) is block-diagonal, one can reorder it into $\left(N_{y}-1\right)^{2}$ decoupled systems of dimension $N_{x}$. Each such system has a lower tridiagonal matrix with a non-zero upper right corner element, and it can be solved in $O\left(N_{x}\right)$ operations.

The fast solver is summarized below. For readability we omit some details mainly associated with reorderings.

1. Divide the data vector $F$ into $N_{x}$ blocks $F_{m}$ of length $\left(N_{y}-1\right)^{2}$, and multiply each block by the matrix $S^{*} \otimes S^{*}$.

2. Reorder the elements of the transformed vector and solve $\left(N_{y}-1\right)^{2}$ systems, each of dimension $N_{x}$, with the matrices

$$
\left(\begin{array}{ccccc}
a & 0 & \ldots & \ldots & -\alpha h_{x} a \\
\Lambda(i, i) & a & 0 & & \\
a & \Lambda(i, i) & a & & \\
& \ddots & \ddots & \ddots & \\
0 & \ldots & a & \Lambda(i, i) & a
\end{array}\right)
$$

where $\Lambda(i, i)$ denotes a diagonal element of $\Lambda$, see (3.11).

3. Reverse the reordering, and multiply each block of length $\left(N_{y}-1\right)^{2}$ by the matrix $S \otimes S$.

It is well known that the 1D DST of a vector of length $N_{y}$ can be computed in $O\left(\log \left(N_{y}\right)\right)$ operations using the FFT. Note that in the implementation of the algorithm the matrix $S^{*} \otimes S^{*}$ in step 1 is never formed; instead the vector $F_{m}$ is reorganized as a square matrix and a 2D DST is computed (similarly in step 3). Therefore, one can see that this algorithm requires $O\left(N_{x} N_{y}^{2} \log \left(N_{y}\right)\right)$ operations for solving the linear system $A U=F$. 


\section{Regularization Parameter Choice}

No matter which regularization method is used for an ill-posed problem, one core problem is how to choose the regularization parameter. The same is the case for the QBV method.

Using the linearity of the problem (1.1)-(1.4), one can subdivide into one wellposed problem and another ill-posed problem only with the nonhomogeneous Neumann data case. This trick has been widely used to deal with the nonhomogeneous ill-posed problems, see, e.g., the appendix in [25].

For the ill-posed problem (1.1)-(1.4) with all data equal to zero except the nonhomogeneous Neumann Cauchy data, a-priori and a-posteriori parameter choice rules have been given in the $[27]^{4}$. Thus, the regularization parameter can be chosen a-posteriori according to the following theorem.

Theorem 2 [27] Let the problem (1.1)-(1.4) be given with $\phi=0, \psi=0$, and $f=0$, and with the nonhomogeneous Neumann data only. Suppose there exists the a-priori bound

$$
\|u(1, \cdot, \cdot)\| \leq E_{1},
$$

and that the condition (1.10) holds. Choose $\tau>1$ such that $0<\tau \delta<\left\|g^{\delta}\right\|$ and the regularization parameter $\alpha=\alpha(\delta)>0$ such that

$$
\left\|v_{x}^{\alpha, \delta}(0, \cdot, \cdot)-g^{\delta}(\cdot, \cdot)\right\|=\tau \delta
$$

Then, for $0<x<1$, we have the following estimate

$$
\left\|u(x, \cdot, \cdot)-v^{\alpha, \delta}(x, \cdot, \cdot)\right\| \leq C E_{1}^{x} \delta^{1-x},
$$

where $C=\left(1+\sqrt{2 \frac{1+(\tau-1)^{2}}{(\tau-1)^{2}}}\right)^{x}(1+\tau)^{1-x}$.

For the convenience of the numerical application, we here do not consider the a-priori choice rule but use the same Morozov's discrepancy principle (4.2) of the a-posteriori parameter choice directly without decomposing the original problem. Some numerical results given later will show that this choice rule is rational and works efficiently.

\section{Numerical implementation and experiments}

In this section, we illustrate the method by solving $2 \mathrm{D}$ and $3 \mathrm{D}$ problems with variable coefficients. To obtain the regularization parameter $\alpha$, we choose $\tau=1.1$ in (4.2), as suggested by Hanke and Hansen [28, 29].

\footnotetext{
${ }^{4}$ In [27], the equation has the coefficient $a=1$, which is not essentially different, since we can divide the coefficients $a, b, c$ by $a$.
} 
All our computations were performed using MATLAB 7.9 on a PC (Intel Core i5 with clock frequency $2 \mathrm{GHz}$ ).

As is customary in the numerical solution of ill-posed test problems, we add normally distributed random perturbations to the data. Thus the perturbed right hand side is computed in MATLAB as $F_{\text {pert }}=F+\epsilon * \operatorname{randn}(\operatorname{size}(\mathrm{F}))$, where the parameter $\epsilon$ determines the magnitude of the perturbation.

In order to compare our preconditioner with the one in [27], we start with the $2 \mathrm{D}$ case, and then consider $3 \mathrm{D}$ problems.

\subsection{Two dimensional case}

Naturally discretization of a 2D problem is less complicated than that in Section 3.1. Consider

$$
\begin{array}{lr}
\left(a u_{x}\right)_{x}+\left(b u_{y}\right)_{y}=f(x, y), & (x, y) \in(0,1) \times(0,1), \\
u(x, 0)=\phi_{1}(x), & x \in[0,1], \\
u(x, 1)=\phi_{2}(x), & x \in[0,1], \\
u(0, y)=\psi(y), & y \in[0,1], \\
u_{x}(0, y)=g(y), & y \in[0,1] .
\end{array}
$$

For this problem, the finite difference discretization of the QBV problems gives a linear system that we denote $\widehat{A} U=F$. To construct the preconditioner $M_{1}$ we replace the variable coefficients $a(x, y), b(x, y)$ by the constants $a, b$ obtained by computing the average over the unit square,

$$
\begin{aligned}
a & :=\int_{0}^{1} \int_{0}^{1} a(x, y) d x d y \\
b & :=\int_{0}^{1} \int_{0}^{1} b(x, y) d x d y
\end{aligned}
$$

The preconditioner becomes

$$
M_{1}=\left(\begin{array}{ccccc}
a I & 0 & \ldots & \ldots & \alpha h_{x} a I \\
T_{1} & a I & 0 & & \\
a I & T_{1} & a I & & \\
& \ddots & \ddots & \ddots & \\
0 & \ldots & a I & T_{1} & a I
\end{array}\right)
$$


where $T_{1}$ is tridiagonal,

$$
T_{1}=\left(\begin{array}{ccccc}
-2(a+b r) & b r & 0 & \cdots & 0 \\
b r & -2(a+b r) & b r & \cdots & 0 \\
& & \ddots & & \\
0 & 0 & \cdots & b r & -2(a+b r)
\end{array}\right) .
$$

Based on the discussion in Section 3.3, we see that the linear system $M_{1} z=y$ can be solved in $N_{x} N_{y} \log \left(N_{y}\right)$ operations using the DST.

In fact, we can also divide the problem into a well-posed problem

$$
\begin{array}{lrl}
\left(a u_{x}^{(1)}\right)_{x}+\left(b u_{y}^{(1)}\right)_{y}=f(x, y), & (x, y) \in(0,1) \times(0,1), \\
u^{(1)}(x, 0)=\phi_{1}(x), & x \in[0,1], \\
u^{(1)}(x, 1)=\phi_{2}(x), & x \in[0,1], \\
u^{(1)}(0, y)=\psi(y), & y \in[0,1], \\
u^{(1)}(1, y)=(1-y) \phi_{1}(1)+y \phi_{2}(1), & y \in[0,1],
\end{array}
$$

and an ill-posed problem

$$
\begin{array}{lr}
\left(a u^{(2)}\right)_{x}+\left(b u_{y}^{(2)}\right)_{y}=0, & (x, y) \in(0,1) \times(0,1), \\
u^{(2)}(x, 0)=0, & x \in[0,1], \\
u^{(2)}(x, 1)=0, & x \in[0,1], \\
u^{(2)}(0, y)=0, & y \in[0,1], \\
u^{(2)}{ }_{x}(0, y)=g(y)-u^{(1)}{ }_{x}(0, y), & y \in[0,1] .
\end{array}
$$

Then $u(x, y)=u^{(1)}(x, y)+u^{(2)}(x, y)$ is the solution of the original problem. For the derivative approximation of the same accuracy $O\left(h_{x}{ }^{2}\right)$ as the general discretization, we give a three-point approximation for $u_{x}$ as $u_{x}(0, y)=(3 u(0, y)-$ $\left.4 u\left(h_{x}, y\right)+u\left(2 h_{x}, y\right)\right) /\left(-2 h_{x}\right)$. Since it needs to compute two problems, the time takes almost twice.

Example 2 Consider an example with the exact solution $u(x, y)=\sin (0.4 x+y)$, which satisfies

$$
\begin{cases}\left((0.4 x+y+1) / 0.16 u_{x}\right)_{x}+\left((0.4 x y+1) u_{y}\right)_{y}=f(x, y), & (x, y) \in(0,1) \times(0,1), \\ u(x, 0)=\sin (0.4 x), & x \in[0,1], \\ u(x, 1)=\sin (0.4 x+1), & x \in[0,1], \\ u(0, y)=\sin (y), & y \in[0,1], \\ u_{x}(0, y)=0.4 \cos (y), & y \in[0,1],\end{cases}
$$



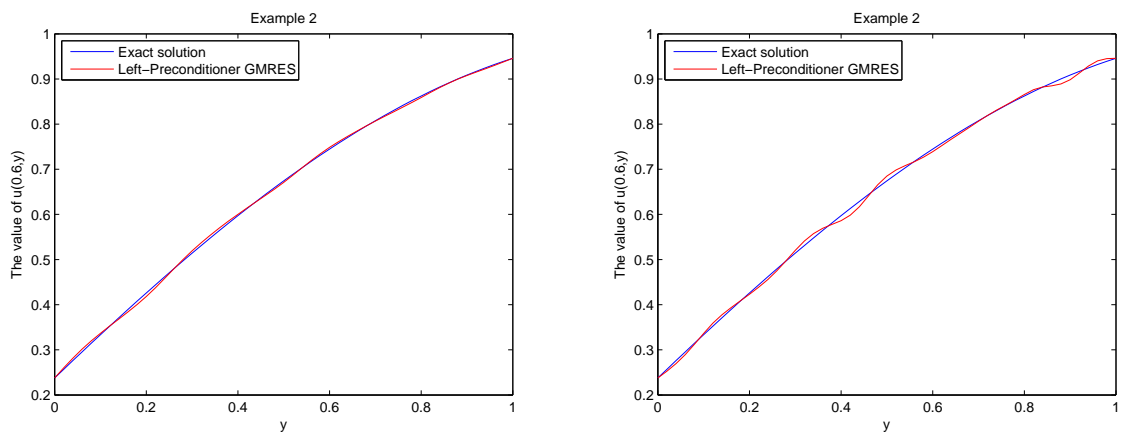

Figure 2: Example 2: Numerical solution computed by left-preconditioned GMRES at $x=0.6$. The regularization parameter was chosen equal to $\alpha=0.01$ (left), and $\alpha=0.001$ (right).

with $f(x, y)=(1+0.4 x) \cos (0.4 x+y)-(0.4 x+y+0.4 x y+2) \sin (0.4 x+y)$.

Discretizing with $N_{x}=100$ and $N_{y}=250$, the $Q B V$ matrix $\widehat{A}$ has dimension $24900 \times 24900$. We solved the linear system $\widehat{A} U=F_{\text {pert }}$ with $\epsilon=10^{-3}$, using GMRES with the left-preconditioner (5.8). GMRES, with residual tolerance $10^{-6}$ converged in 12 iterations and 10.9 seconds. To study the sensitivity to the regularization parameter $\alpha$, we chose several values and compared the solutions. The results for two representative values are illustrated in Figure 2.

In [27] we solved a 2D problem with constant coefficients, using left-preconditioned GMRES, where the preconditioner was a circulant matrix. There we performed 30 iterations, restarted, and then performed another 25 iterations before the procedure converged. The same convergence criterion was used.

From the results illustrated in Figure 2, we see that the solution is not very sensitive to the choice of regularization parameter $\alpha$.

\subsection{Three dimensional case}

Now let us use the same idea for a 3D problem to see if our method works.

To see if the QBV method works well for the case of constant coefficients, we use MATLAB 7.9 to do some tests in this subsection. We divide the interval of $x$ into $N_{x}=50$ segments and the interval of $y_{1}$ and $y_{2}$ directions both into $N_{y}=100$ segments. The coefficient matrix $A$ in (3.9) is a large sparse matrix having the size $490050 \times 490050$. In (3.10), $T_{1}=\operatorname{tridiag}(r * c,-2 *(a+r * b+r * c), r * c)$ and $T_{2}=\operatorname{tridiag}(r * b, 0, r * b)$, where $r=\left(h_{x} / h_{y}\right)^{2}$. 
Example 3 We consider a homogeneous case with an exact solution $u\left(x, y_{1}, y_{2}\right)=$ $\sinh (2 \sqrt{2} \pi x) \sin \left(2 \pi y_{1}\right) \sin \left(2 \pi y_{2}\right)$, i.e.,

$$
\begin{cases}u_{x x}+u_{y_{1} y_{1}}+u_{y_{2} y_{2}}=0, & \left(x, y_{1}, y_{2}\right) \in(0,1)^{3}, \\ u\left(x, 0, y_{2}\right)=0, & \left(x, y_{2}\right) \in[0,1]^{2}, \\ u\left(x, 1, y_{2}\right)=0, & \left(x, y_{2}\right) \in[0,1]^{2}, \\ u\left(x, y_{1}, 0\right)=0, & \left(x, y_{1}\right) \in[0,1]^{2}, \\ u\left(x, y_{1}, 1\right)=0, & \left(x, y_{1}\right) \in[0,1]^{2}, \\ u\left(0, y_{1}, y_{2}\right)=0, & \left(y_{1}, y_{2}\right) \in[0,1]^{2}, \\ u_{x}\left(0, y_{1}, y_{2}\right)=2 \sqrt{2} \pi \sin \left(2 \pi y_{1}\right) \sin \left(2 \pi y_{2}\right), & \left(y_{1}, y_{2}\right) \in[0,1]^{2} .\end{cases}
$$

Example 4 We consider a nonhomogeneous case with an exact solution $u\left(x, y_{1}, y_{2}\right)=$ $\exp \left(x+y_{1}+y_{2}\right)$, i.e.,

$$
\begin{cases}u_{x x}+u_{y_{1} y_{1}}+u_{y_{2} y_{2}}=3 \exp \left(x+y_{1}+y_{2}\right), & \left(x, y_{1}, y_{2}\right) \in(0,1)^{3}, \\ u\left(x, 0, y_{2}\right)=\exp \left(x+y_{2}\right), & \left(x, y_{2}\right) \in[0,1]^{2}, \\ u\left(x, 1, y_{2}\right)=\exp \left(x+1+y_{2}\right), & \left(x, y_{2}\right) \in[0,1]^{2}, \\ u\left(x, y_{1}, 0\right)=\exp \left(x+y_{1}\right), & \left(x, y_{1}\right) \in[0,1]^{2}, \\ u\left(x, y_{1}, 1\right)=\exp \left(x+y_{1}+1\right), & \left(x, y_{1}\right) \in[0,1]^{2}, \\ u\left(0, y_{1}, y_{2}\right)=\exp \left(y_{1}+y_{2}\right), & \left(y_{1}, y_{2}\right) \in[0,1]^{2}, \\ u_{x}\left(0, y_{1}, y_{2}\right)=\exp \left(y_{1}+y_{2}\right), & \left(y_{1}, y_{2}\right) \in[0,1]^{2} .\end{cases}
$$

Figure 3 shows the results of Example 3 for $x_{0}=0.5$ with $\epsilon=10^{-3} .5$ Figure $3(\mathrm{a})$ is the exact solution and Figure $3(\mathrm{~b})$ is the approximation whose regularization parameter $\alpha=4.68753 \times 10^{-7}$ chosen by Morozov's discrepancy principle (4.2). Figure 4 illustrates the solutions of Example 4 for $x_{0}=0.4$ with $\epsilon=10^{-2}$. Figure 4(a) is the exact solution and Figure $4(\mathrm{~b})$ is the approximated solution with $\alpha=3 \times 10^{-5}$. From the tests, it is seen that our method works efficiently.

On the basis of the above fast solver for constant coefficient case, in the following, we will consider the variable coefficient case. Similar as the preconditioner $\widetilde{M}_{1}$, we construct a preconditioner $\widetilde{M}_{2}$ for $3 \mathrm{D}$ as following

$$
\widetilde{M}_{2}=\left(\begin{array}{ccccc}
\widetilde{a} I & 0 & \ldots & \ldots & \alpha h_{x} \widetilde{a} I \\
\widetilde{T}_{2} & \widetilde{a} I & 0 & & \\
\widetilde{a} I & \widetilde{T}_{2} & \widetilde{a} I & & \\
& \ddots & \ddots & \ddots & \\
0 & \ldots & \widetilde{a} I & \widetilde{T}_{2} & \widetilde{a} I
\end{array}\right)
$$

\footnotetext{
${ }^{5}$ For a vector $v$, we construct the corresponding noisy data by adding a distributed perturbation to the data $v$, i.e., $v^{\delta}=v+\epsilon \operatorname{randn}(\operatorname{size}(v))$.
} 

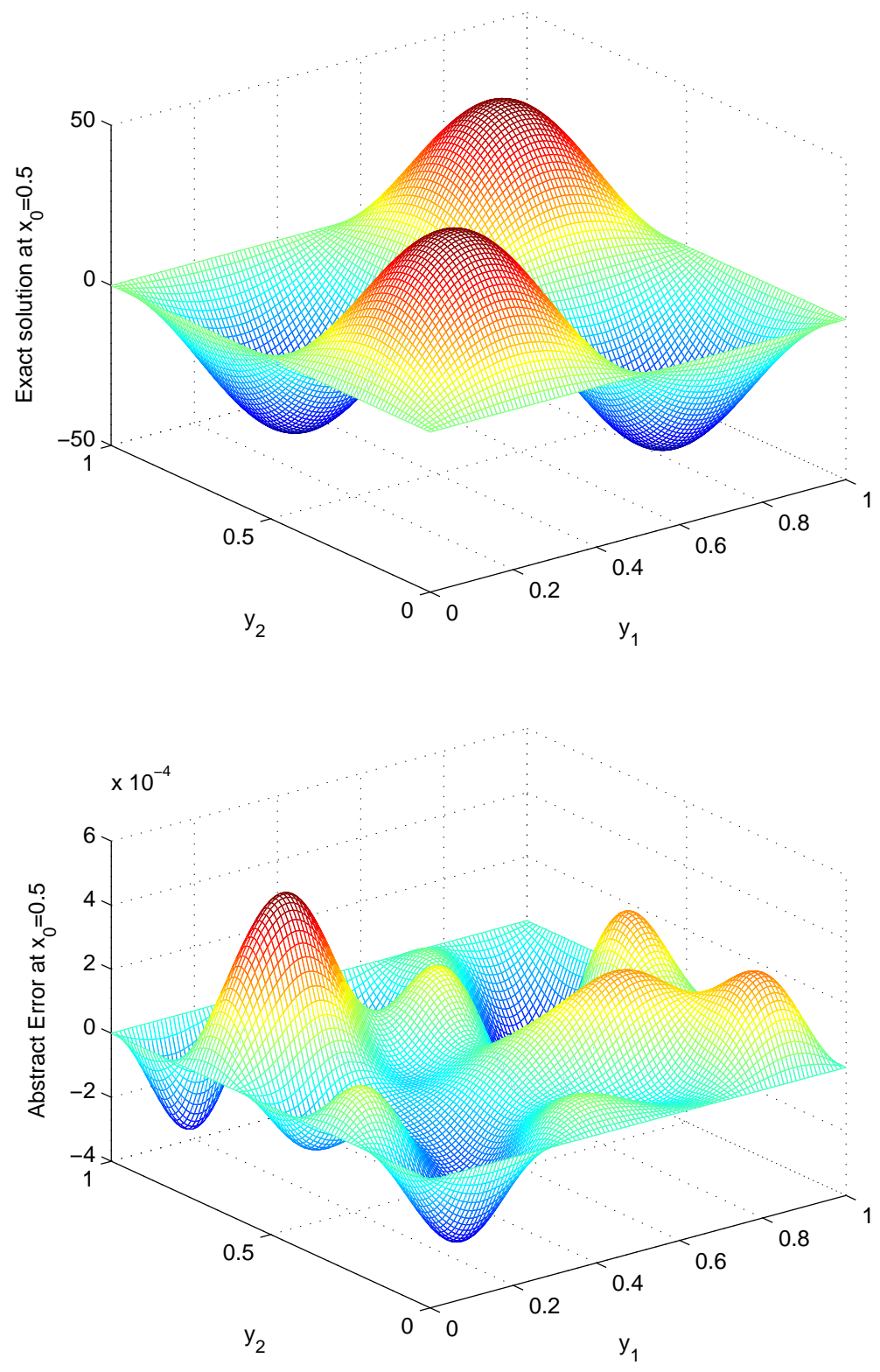

Figure 3: Example 3 at $x_{0}=0.5$ : (a) Exact solution; (b) The error between the exact solution and approximation with $\epsilon=10^{-3}$ and $\alpha=4.68753 \times 10^{-7}$. 

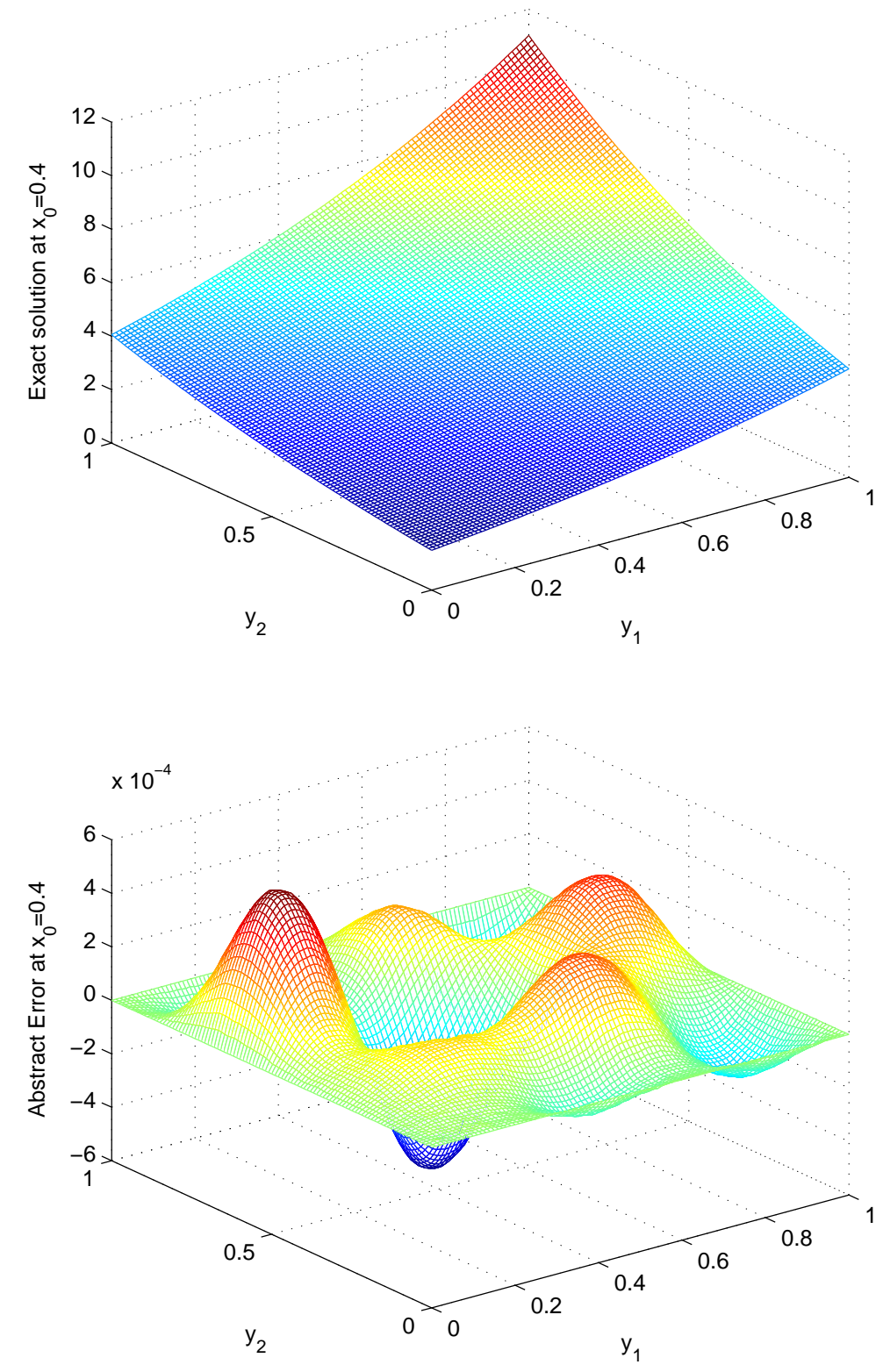

Figure 4: Example 4 at $x_{0}=0.4$ : (a) Exact solution; (b) The error between the exact solution and approximation with $\epsilon=10^{-2}$ and $\alpha=3 \times 10^{-5}$. 
where the block

$$
\widetilde{T}_{2}=\widetilde{T}_{2}^{2} \otimes I+I \otimes \widetilde{T}_{2}^{1}
$$

and $\widetilde{T}_{2}^{1}=\operatorname{tridiag}(r \widetilde{c},-2(\widetilde{a}+r \widetilde{b}+r \widetilde{c}), r \widetilde{c}), \widetilde{T}_{2}^{2}=\operatorname{tridiag}(r \widetilde{b}, 0, r \widetilde{b})$ with

$$
\begin{aligned}
\widetilde{a} & :=\int_{0}^{1} \int_{0}^{1} \int_{0}^{1} a\left(x, y_{1}, y_{2}\right) d x d y_{1} d y_{2} \\
\widetilde{b} & :=\int_{0}^{1} \int_{0}^{1} \int_{0}^{1} b\left(x, y_{1}, y_{2}\right) d x d y_{1} d y_{2} \\
\widetilde{c} & :=\int_{0}^{1} \int_{0}^{1} \int_{0}^{1} c\left(x, y_{1}, y_{2}\right) d x d y_{1} d y_{2} .
\end{aligned}
$$

We also give two examples (Example 1 and Example 5) to verify our method. For both examples, we have a finite difference grid of dimension $50 \times 100 \times 100$, i.e., the matrix $\widetilde{A}$ in $(3.3)$ has dimension $490050 \times 490050$. Using the LeftPreconditioned GMRES method with the preconditioner $\widetilde{M}_{2}$, solving the 3D Examples 1 and 5 only needs 15 and 13 iteration steps. The total time spent in the function GMRES for Examples 1 and 5 is 86 and 69 seconds, respectively. This means we can solve the $3 \mathrm{D}$ problems within reasonable time. The results given in Figures 5 and 6 show that our method works efficiently and the preconditioner $\widetilde{M}_{2}$ is good.

Example 5 We consider a case with $a\left(x, y_{1}, y_{2}\right)=x y_{1} y_{2}+1, b\left(x, y_{1}, y_{2}\right)=x\left(y_{1}+\right.$ $\left.y_{2}\right)+1, c\left(x, y_{1}, y_{2}\right)=x+y_{1}+y_{2}+1$, and an exact solution $u\left(x, y_{1}, y_{2}\right)=\exp (x+$ $\left.y_{1}+y_{2}\right)$, i.e.,

$$
\begin{cases}\left(a u_{x}\right)_{x}+\left(b u_{y_{1}}\right)_{y_{1}}+\left(c u_{y_{2}}\right)_{y_{2}}=f\left(x, y_{1}, y_{2}\right), & \left(x, y_{1}, y_{2}\right) \in(0,1)^{3}, \\ u\left(x, 0, y_{2}\right)=\exp \left(x+y_{2}\right), & \left(x, y_{2}\right) \in[0,1]^{2}, \\ u\left(x, 1, y_{2}\right)=\exp \left(x+1+y_{2}\right), & \left(x, y_{2}\right) \in[0,1]^{2}, \\ u\left(x, y_{1}, 0\right)=\exp \left(x+y_{1}\right), & \left(x, y_{1}\right) \in[0,1]^{2}, \\ u\left(x, y_{1}, 1\right)=\exp \left(x+y_{1}+1\right), & \left(x, y_{1}\right) \in[0,1]^{2}, \\ u\left(0, y_{1}, y_{2}\right)=\exp \left(y_{1}+y_{2}\right), & \left(y_{1}, y_{2}\right) \in[0,1]^{2}, \\ u_{x}\left(0, y_{1}, y_{2}\right)=\exp \left(y_{1}+y_{2}\right), & \left(y_{1}, y_{2}\right) \in[0,1]^{2},\end{cases}
$$

with $f\left(x, y_{1}, y_{2}\right)=x y_{1} y_{2}+x y_{1}+x y_{2}+y_{1} y_{2}+2 x+y_{1}+y_{2}+4$.

\section{Conclusion}

We have proposed a QBV method for solving a Cauchy problem for a 3D elliptic PDE with variable coefficients. Some stability error estimates are given. A large sparse linear system obtained from the QBV problem has been dealt with by a Left-Preconditioned GMRES method. A good preconditioner is constructed and 

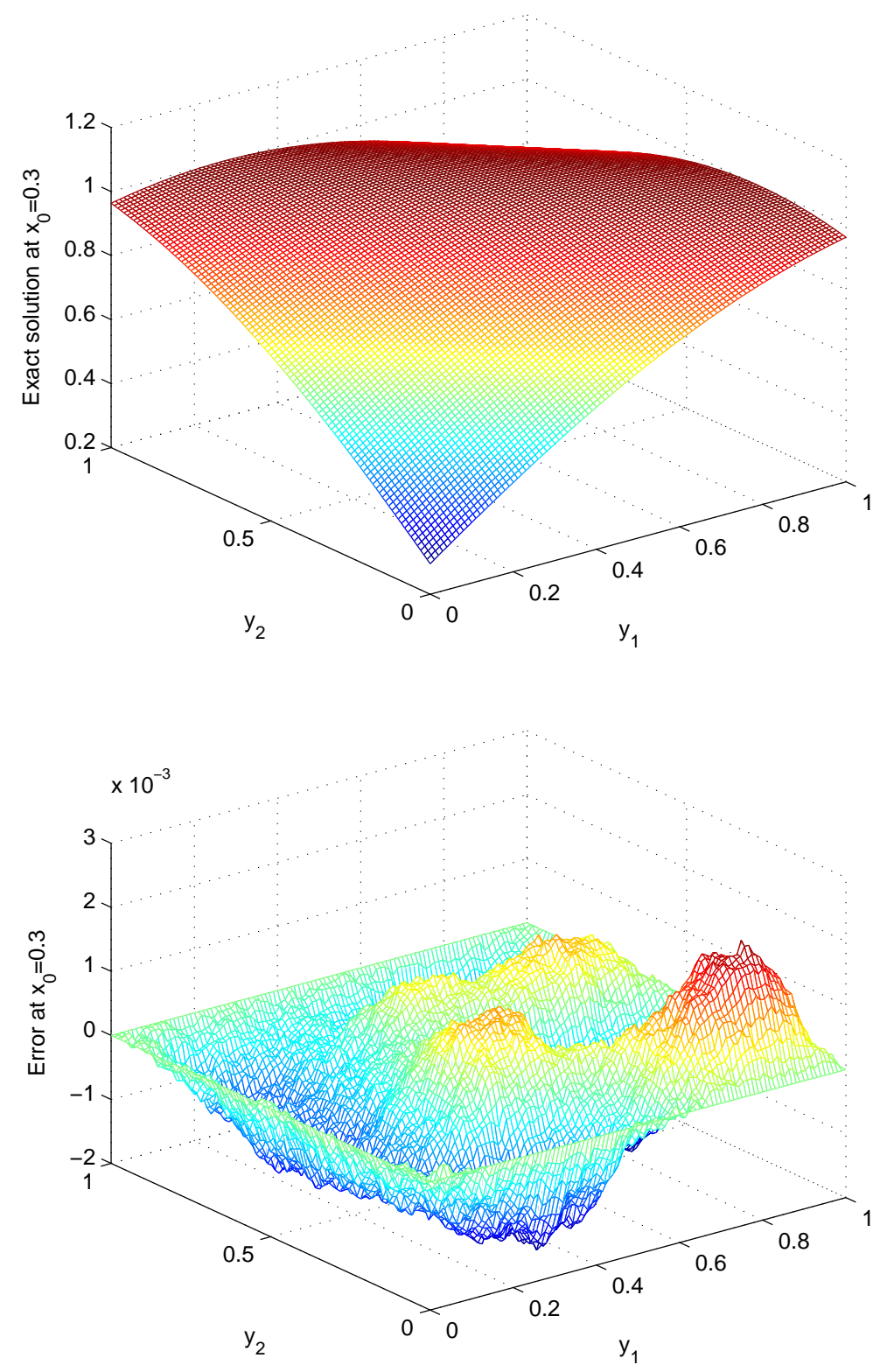

Figure 5: Example 1: The results solved by the Left-Preconditioned GMRES method at $x_{0}=0.3$ with $\epsilon=10^{-3}$ and $\alpha=0.001$. (a). The exact solution (b). The error between the exact solution and approximation 

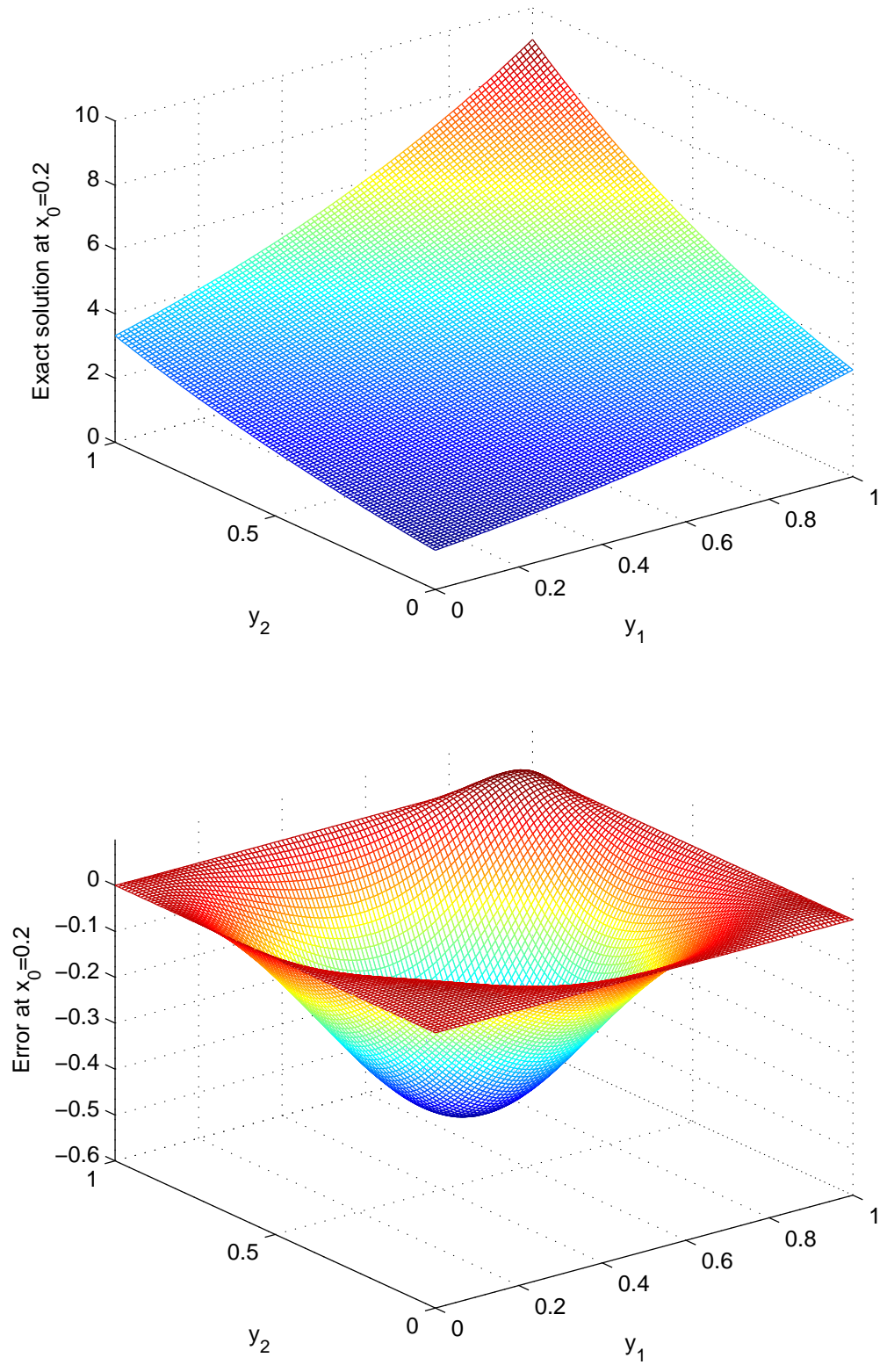

Figure 6: Example 5: The results solved by the Left-Preconditioned GMRES method at $x_{0}=0.2$ with $\epsilon=10^{-3}$ and $\alpha=0.001$. (a). The exact solution (b). The error between the exact solution and approximation 
a fast solver using the FFT is given to solve the preconditioned system. When using the fast solver, time and memory are also saved. Numerical results show that the method works effectively. The proposed method is effective for the rectangular parallelepiped using FDM. It should be more interesting to construct a good preconditioner for the general domain using finite element method, which is our future work.

\section{Appendix: Proof of Theorem 1}

Here we just outline the framework. One can refer to paper [24] for more detail.

We use the logarithmic convexity method to obtain the stability result Theorem 1. Therefore, we want to get an energy functional. After some complicated computation, one can use the similar technique given in [24] to get the following energy functional as

$$
F(\beta)=\int_{D_{\beta}}(\beta-x) a w\left(\beta w+w_{x}\right) d x d y_{1} d y_{2},
$$

where the domain $D_{\beta}$ is defined by $(2.5)$, and $w$ satisfies the following problem

$$
\begin{array}{lr}
\lambda a\left(\lambda w+w_{x}\right)+\left(a\left(\lambda w+w_{x}\right)\right)_{x}+\left(b w_{y_{1}}\right)_{y_{1}}+\left(c w_{y_{2}}\right)_{y_{2}}=0, & \left(x, y_{1}, y_{2}\right) \in(0, \beta) \times \Omega, \\
w\left(x, 0, y_{2}\right)=w\left(x, 1, y_{2}\right)=w\left(x, y_{1}, 0\right)=w\left(x, y_{1}, 1\right)=0, & x \in[0, \beta],\left(y_{1}, y_{2}\right) \in \partial \Omega, \\
w\left(0, y_{1}, y_{2}\right)=0, & \left(y_{1}, y_{2}\right) \in \bar{\Omega}, \\
\lambda w\left(0, y_{1}, y_{2}\right)+w_{x}\left(0, y_{1}, y_{2}\right)=g\left(y_{1}, y_{2}\right), & \left(y_{1}, y_{2}\right) \in \bar{\Omega} .
\end{array}
$$

Here $\lambda \geq 0$ is to be chosen later.

From some derivation, one can get the following three Lemmas:

Lemma 1 Assume that $w$ satisfies the above problem, and that for all $\left(x, y_{1}, y_{2}\right) \in$ $D_{1}, a_{x}$ satisfies (1.5). Then, if $\lambda$ is chosen such that $\lambda \geq a_{2} /\left(2 a_{0}\right)$, the following estimates hold:

$$
\frac{1}{2} \int_{D_{\beta}} a w^{2} d x d y_{1} d y_{2} \leq F(\beta) \leq \tilde{c} \int_{D_{\beta}} a w^{2} d x d y_{1} d y_{2}, \tilde{c}=\frac{1}{2}+\lambda+\frac{a_{1}}{2 a_{0}},
$$

and

$$
F F^{\prime \prime}-\left(F^{\prime}\right)^{2} \geq F\left[\int_{D_{\beta}}\left(b w_{y_{1}}^{2}+c w_{y_{2}}^{2}-a\left(\lambda w+w_{x}\right)^{2}\right) d x d y_{1} d y_{2}-2 \lambda F^{\prime}\right] .
$$

Lemma 2 The following identity holds:

$$
\int_{D_{\beta}}\left(b w_{y_{1}}^{2}+c w_{y_{2}}^{2}-a\left(\lambda w+w_{x}\right)^{2}\right) d x d y_{1} d y_{2}
$$




$$
\begin{aligned}
= & -2 \lambda \int_{D_{\beta}}(\beta-x)\left(b w_{y_{1}}^{2}+c w_{y_{2}}^{2}-a\left(\lambda w+w_{x}\right)^{2}\right) d x d y_{1} d y_{2} \\
& +\int_{D_{\beta}}(\beta-x)\left(b_{x} w_{y_{1}}^{2}+c_{x} w_{y_{2}}^{2}+a_{x}\left(\lambda w+w_{x}\right)^{2}\right) d x d y_{1} d y_{2} \\
& -\beta \int_{0}^{1} \int_{0}^{1}\left(a\left(\lambda w+w_{x}\right)^{2}\right)\left(0, y_{1}, y_{2}\right) y_{1} d y_{2} .
\end{aligned}
$$

Lemma 3 Assume that for all $\left(x, y_{1}, y_{2}\right) \in D_{1}, a_{x}, b_{x}$ and $c_{x}$ satisfy (1.5). Let

$$
\tilde{c_{0}}=\max \left(\frac{a_{1}}{a_{0}}, \frac{b_{1}}{b_{0}}, \frac{c_{1}}{c_{0}}\right) \text {. }
$$

Then

$$
\int_{D_{\beta}}(\beta-x)\left(b_{x} w_{y_{1}}^{2}+c_{x} w_{y_{2}}^{2}+a_{x}\left(\lambda w+w_{x}\right)^{2}\right) d x d y_{1} d y_{2} \geq-\tilde{c_{0}}\left(F^{\prime}+2 \lambda F\right),
$$

and

$$
-\int_{D_{\beta}}(\beta-x)\left(b w_{y_{1}}^{2}+c w_{y_{2}}^{2}-a\left(\lambda w+w_{x}\right)^{2}\right) d x d y_{1} d y_{2} \geq-\left(F^{\prime}+2 \lambda F\right) .
$$

According to the above lemmas, we can get the following important result:

Theorem 3 Assume that

$$
\lambda \geq \max \left(\frac{a_{2}}{2 a_{0}}, \frac{a_{1}}{a_{0}}\right),
$$

and that the coefficients satisfy (1.5). Then the stability functional satisfies the inequality

$$
F F^{\prime \prime}-\left(F^{\prime}\right)^{2} \geq-K_{1} F F^{\prime}-K_{2} F^{2},
$$

where $K_{1}$ and $K_{2}$ satisfy (2.6).

Now we define

$$
\sigma=e^{-K_{1} \beta},
$$

and regard $F$ as a function of $\sigma$. We introduce the auxiliary function

$$
G(\sigma)=\log \left[F(\beta(\sigma)) \sigma^{-K_{2} / K_{1}^{2}}\right] .
$$

Thus Theorem 3 shows that $G(\sigma)$ is convex on the interval $\sigma_{1} \leq \sigma \leq 1$, with $\sigma_{1}=e^{-K_{1}}$. Therefore,

$$
G(\sigma) \leq \frac{\sigma-\sigma_{1}}{1-\sigma_{1}} G(1)+\frac{1-\sigma}{1-\sigma_{1}} G\left(\sigma_{1}\right)
$$

which is equivalent to

$$
F(\beta) \leq e^{(-\beta+\nu \beta) K_{2} / K_{1}}[F(0)]^{1-\nu(\beta)}[F(1)]^{\nu(\beta)},
$$

where $\nu(\beta)$ is defined by $(2.7)$.

Now it is easy to prove Theorem 1 . 


\section{Acknowledgement}

We are indebted to Dr. Fredrik Berntsson for his support in numerical aspects.

\section{References}

[1] J. Aarão, B.H. Bradshaw-Hajek, S.J. Miklavcic and D.A. Ward, The extended-domain eigenfunction method for solving elliptic boundary value problems with annular domains, J. Phys. A: Math. Theor., 43(2010), $185202(18 \mathrm{pp})$.

[2] L.Š. Abdulkerimov, Regularization of an ill-posed Cauchy problem for evolution equations in a Banach space, Azerbaidzan. Gos. Univ. Ucen. Zap. Fiz. Mat., 1(1977), 32-36 (MR0492645) (in Russian).

[3] G. Alessandrini, L. Rondi, E. Rosset and S. Vessella, The stability for the Cauchy problem for elliptic equations, Inverse Problems, 25(2009), 123004 (47pp).

[4] K.A. Ames and L.E. Payne, Asymptotic for tow regularizations of the Cauchy problem for the backward heat equation, Math. Models Methods Appl. Sci., 8(1998), 187-202.

[5] K.A. Ames, L.E. Payne and P.W. Schaefer, Energy and pointwise bounds in some non-standard parabolic problems, Proc. Roy. Soc. Edinburgh Sect. A, 134(2004), 1-9.

[6] S. Andrieux, T.N. Baranger and A. Ben Abda, Solving Cauchy problems by minimizing an energy-like functional, Inverse Problems, 22(2006), 115-133.

[7] S.R. Arridge, Optical tomography in medical imaging, Inverse Problems, 15(1999), R41-R93.

[8] M. Azaïez, F.B. Belgacem and H. El Fekih, On Cauchy problem: II. Completion, regularization and approximation, Inverse Problems, 22(2006), 13071336 .

[9] F.B. Belgacem, Why is the Cauchy problem severely ill-posed? Inverse Problems, 23(2007), 823-836.

[10] L. Borcea, Electrical impedance tomography, Inverse Problems, 18(2002), R99-R136.

[11] P. Brianzi, P. Favati, O. Menchi and F. Romani, A framework for studying the regularizing properties of Krylov subspace methods, Inverse Problems, 22(2006), 1007-021. 
[12] A.M. Bruaset, A survey of preconditioned iterative methods, Pitman Research Notes in Mathematics Series, Longman Scientific \& Technical, 1995.

[13] D. Calvetti, B. Lewis and L. Reichel, GMRES-type methods for inconsistent systems, Linear Alg. Appl., 316(2000), 157-169.

[14] D. Calvetti, B. Lewis and L. Reichel, GMRES, L-curves, and discrete ill-posed problems, BIT, 42(2002), 44-65.

[15] D. Calvetti, B. Lewis and L. Reichel, On the regularizing properties of the GMRES method, Numer. Math., 91(2002), 605-625.

[16] H. Cheng, X.L. Feng and C.L. Fu, A mollification regularization method for the Cauchy problem of an elliptic equation in a multi-dimensional case, Inverse Problems in Science and Engineering, First published on: 29 June 2010 (iFirst).

[17] D. Colton and R. Kress, Inverse acoustic and electromagnetic scattering theory, Springer, 1998(2nd edition).

[18] H. Cheng, C.L. Fu and X.L. Feng, Determining surface heat flux in the steady state for the Cauchy problem for the Laplace equation, Applied Mathematics and Computation, 211(2009), 374-382.

[19] J. Cheng and M. Yamamoto, One new strategy for a priori choice of regularizing parameters in Tikhonov regularization, Inverse Problems, 16(2000), L31-L38.

[20] P.J. Davis, Circulant Matrices, AMS Chelsea Publishing, 1979.

[21] M. Denche and K. Bessila, Quasi-boundary Value Method for Non-well Posed Problem for a Parabolic Equation with Integral Boundary Condition, Math. Probl. Eng., 7(2001), 129-145.

[22] M. Denche and K. Bessila, A modified quasi-boundary value method for illposed problems, J. Math. Anal. Appl., 301(2005), 419-426.

[23] H. Egger, Y. Heng, W. Marquardt and A. Mhamdi, Efficient solution of a three-dimensional inverse heat conduction problem in pool boiling, Inverse Problems, 25(2009), 095006.

[24] L. Eldén and F. Berntsson, A stability estimate for a Cauchy problem for an elliptic partial differential equation, Inverse Problems, 21(2005), 1643-1653.

[25] L. Eldén and V. Simoncini, A numerical solution of a Cauchy problem for an elliptic equation by Krylov subspaces, Inverse Problems, 25(2009), 065002 (22pp). 
[26] L. Eldén and V. Simoncini, Solving ill-posed linear systems with GMRES and a singular preconditioner, SIAM J. Matrix Anal. Appl. 33(2012) 1369-1394.

[27] X.L. Feng, L. Eldén and C.L. Fu, A quasi-boundary-value method for the Cauchy problem for elliptic equations with nonhomogeneous Neumann data, Journal of Inverse and Ill-Posed Problems, 18(2010), 617-645.

[28] M. Hanke, Conjugate Gradient Type Methods for Ill-posed Problems (Harlow: Longman Scientific and Technical), 1995.

[29] M. Hanke and P.C. Hansen, Regularization methods for large-scale problems, Surveys Math. Industry, 3(1993), 253-315.

[30] M. Hanke and J. Nagy, Restoration of atmospherically blurred images by symmetric indefinite conjugate gradient techniques, Inverse Problems, 12(1996), $157-73$.

[31] P.C. Hansen and T.K. Jensen, Smoothing-norm preconditioning for regularizing minimum-residual methods, SIAM Journal on Matrix Analysis and Applications, 29(2006), 1-14.

[32] D.N. Hào, V.D. Nguyen and H. Sahli, A non-local boundary value problem method for parabolic equations backward in time, J. Math. Anal. Appl., 345(2008), 805-815.

[33] D.N. Hào and N.V. Duc, Regularization of parabolic equations backward in time by a non-local boundary value problem method, IMA J. Appl. Math, 2009, 1-25, doi:10.1093/imamat/hxp026.

[34] D.N. Hào, N.V. Duc and D. Lesnic, A non-local boundary value problem method for the Cauchy problem for elliptic equations, Inverse Problems, $25(2009), 055002(27 \mathrm{pp})$.

[35] R.W. Hockney, A Fast Direct Solution of Poisson's Equation Using Fourier Analysis, J. ACM., 12(1965), 95-113.

[36] V. Isakov, Inverse Source Problems (Mathematical Surveys and Monographs vol 34) (Providence, RI: American Mathematical Society), 1990.

[37] V. Isakov, Inverse Problems for Partial Differential Equations (Applied Mathematical Sciences vol 127) 2nd edn (New York: Springer), 2006.

[38] T.K. Jensen and P.C. Hansen, Iterative regularization with minimumresidual methods, BIT Numerical Mathematics, 47(2007), 103-120.

[39] M. Kilmer and G.W. Stewart, Iterative regularization and MINRES, SIAM J. Matrix Anal. Appl., 21(1999), 613-628. 
[40] V. Kozlov, V. Maz'ya and A.F. Fomin, An iterative method for solving the Cauchy problem for elliptic equations, Comput. Math. Math. Phys., 31(1992), $45-52$.

[41] M.M. Lavrentiev, Some Improperly Posed Problems of Mathematical Physics (New York: Springer-Verlag), 1967.

[42] M.M. Lavrent'ev, V.G. Romanov and S.P. Šišatskiı̌, Nekorrektnye Zadachi Matematicheskoi Fiziki i Analiza (Moscow: Nauka), 1980.

M.M. Lavrentev, V.G. Romanov and S.P. Šišatskiǐ, Problemi non ben posti in fisica matematica e analisi Pubblicazioni dellIstituto di Analisi Globale e Applicazioni 12 (Firenze: IAGA) (Italian Translation), 1983.

M.M. Lavrentev, V.G. Romanov and S.P. Šišatskiǐ, Ill-Posed Problems of Mathematical Physics and Analysis (Translations of Mathematical Monographs vol 64) (Providence, RI: American Mathematical Society) (Engl. Transl.), 1986.

[43] I.V. Mel'nikova, Regularization of ill-posed differential problems, Siberian Math. J., 33(1992), 289-298.

[44] L.E. Payne, On a priori bounds in the Cauchy problem for elliptic equations, SIAM J. Math. Anal., 1(1970), 82-9.

[45] L.E. Payne, Improperly Posed Problems in Partial Differential Equations (Philadelphia, PA: Society for Industrial and Applied Mathematics), 1975.

[46] L.E. Payne, Improved stability estimates for classes of illposed Cauchy problems, Appl. Anal., 19(1985), 63-4.

[47] Z. Qian, C.L. Fu and Z.P. Li, Two regularization methods for a Cauchy problem for the Laplace equation, J. Math. Anal. Appl., 338(2008), 479-489.

[48] C.Y. Qiu and C.L. Fu, Wavelets and regularization of the Cauchy problem for the Laplace equation, J. Math. Anal. Appl., 338(2008), 1440-1447.

[49] Z. Ranjbar, Numerical Solution of Ill-posed Cauchy Problems for Parabolic Equations, PhD Thesis, Department of Mathematics, Linköping University, 2010.

[50] Z. Ranjbar and L. Eldén, Solving an ill-posed Cauchy problem for a $2 \mathrm{~d}$ parabolic PDE with variable coefficients using a preconditioned GMRES Method, Technical Report LiTH-MAT-R-2010/03-SE, Department of Mathematics, Linköping University, 2010. Submitted.

[51] H.J. Reinhardt, H. Han and D.N. Hào, Stability and regularization of a discrete approximation to the Cauchy problem for Laplace's equation, SIAM J. NUMER. ANAL., 36(1999), 890-90551. 
[52] Y. Saad, Iterative Methods for Sparse Linear Systems, 2nd ed. SIAM, Philadelphia, 2003.

[53] Y. Saad and M.H. Schultz, GMRES: A generalized minimal residual algorithm for solving nonsymmetric linear systems, SIAM J. Sci. Stat. Comput., 7(1986), 856-869.

[54] R.E. Showalter, Cauchy Problem for hyper-parabolic partial differential equations, "Trends in the Theory and practice of Non-Linear Analysis", Elsevier, 1983.

[55] G.D. Smith, Numerical solution of partial differential equations: finite difference methods, Engineering Analysis with Boundary Elements, Oxford Applied Mathematics and Computing Science Series I, 1985.

[56] Paul N. Swarztrauber, The Methods of Cyclic Reduction, Fourier Analysis and the FACR Algorithm for the Discrete Solution of Poisson's Equation on a Rectangle, SIAM Review, 19(1977), 490-501.

[57] U. Tautenhahn, Optimal stable solution of Cauchy problems of elliptic equations, J. Analysis and its Applications, 15(1996), 961-984.

[58] P.N. Vabishchevich, Numerical solution of nonlocal elliptic problems, Izv. Vyssh. Uchebn. Zaved. Mat., 1983, 13-19 (in Russian).

[59] P.N. Vabishchevich and A.Y. Denisenko, Regularization of nonstationary problems for elliptic equations, J. Eng. Phys. Thermophys., 65(1993), 11951199.

[60] P.N. Vabishchevich and P.A. Pulatov, A method of numerical solution of the Cauchy problem for elliptic equations, Vestnik Moskov. Univ. Ser. XV Vychisl. Mat. Kibernet., 1984, 3-8 (in Russian).

[61] T. Wei, Y.C. Hon and L. Ling, Method of fundamental solutions with regularization techniques for Cauchy problems of elliptic operators, Engineering Analysis with Boundary Elements, 31(2007), 373-385. 